\title{
Matrix metalloproteinase 14 and 19 expression is associated with thoracic aortic aneurysms
}

\author{
Veronica Jackson, MD, ${ }^{\mathrm{a}}$ Therese Olsson, BMA, ${ }^{\mathrm{b}}$ Sanela Kurtovic, $\mathrm{PhD},{ }^{\mathrm{b}}$ Lasse Folkersen, MSc, ${ }^{\mathrm{b}}$ \\ Valentina Paloschi, MSc, ${ }^{\mathrm{b}}$ Dick Wågsäter, $\mathrm{PhD},{ }^{\mathrm{b}}$ Anders Franco-Cereceda, $\mathrm{MD}, \mathrm{PhD},{ }^{\mathrm{a}}$ and \\ Per Eriksson, $\mathrm{PhD}^{\mathrm{b}}$
}

\begin{abstract}
Objective: It is hypothesized that an altered turnover of extracellular matrix mediated by matrix metalloproteinases (MMPs) is present in thoracic aortic aneurysms. Here, we analyzed the occurrence of MMPs and MMP inhibitors in ascending aortic aneurysms in patients with bicuspid and tricuspid aortic valves.
\end{abstract}

\begin{abstract}
Methods: Expression of 23 MMPs and their inhibitors was measured in aortic intima/media and adventitia in 109 patients (40 tricuspid, 69 bicuspid, 68 with aortic diameter $\geq 4.5 \mathrm{~cm}$, and 41 with $\leq 4.0 \mathrm{~cm}$ ) using Affymetrix Exon arrays (Affymetrix, Santa Clara, Calif). Gene expression was confirmed by quantitative real-time polymerase chain reaction. Principal components analysis was used to study differences in gene expression. Immunohistochemistry was used to study protein expression.
\end{abstract}

Results: We detected messenger RNA expression for gelatinases (MMP2 and MMP9), stromelysin 3 (MMP11), all membrane bound MMPs (MMP14, MMP15, MMP16, MMP17, MMP24, MMP25), MMP19, MMP21, and MMP28 in ascending aorta. No expression of collagenases was detected. Principal components analysis showed that changes in mRNA expression between dilated and nondilated aorta were mainly detected in patients with tricuspid aortic valves. MMP14 and MMP19 showed higher expression in dilated aortas and MMP19 expression correlated positively to maximal aortic diameter in patients with tricuspid aortic valves ( $\mathrm{Rho}=0.61, P=.004$, and Rho $=0.57, P=.008$, using raw and body surface area-corrected aortic diameter, respectively). Immunohistochemical staining demonstrated increased medial expression of MMP14 and MMP19 in dilated aorta.

Conclusions: The present study identifies MMP14 and MMP19 as proteolytic enzymes potentially involved in aneurysm formation in the ascending aorta of patients with tricuspid aortic valves. (J Thorac Cardiovasc Surg 2012;144:459-66)

A bicuspid aortic valve (BAV) is present in $1 \%$ to $2 \%$ of the population in the Western world. ${ }^{1}$ The presence of BAV predisposes for progressive dilatation of the ascending aorta and thereby constitutes an increased risk for aortic aneurysm and/or dissection. ${ }^{2}$ The etiology of the aortic dilatation associated with BAV is still debated, ${ }^{3}$ and it has been suggested that the dilatation is the result of altered hemodynamic factors, that is, owing to abnormal valvular morphologic and pathologic conditions. ${ }^{4}$ The idea of an intrinsic weakness of the aortic wall as the cause of aneurysm

From the Cardiothoracic Surgery Unit, Department of Molecular Medicine and Surgery, and the Atherosclerosis Research Unit, Center for Molecular Medicine, Department of Medicine, ${ }^{\mathrm{b}}$ Karolinska Institutet and Karolinska University Hospital, Stockholm, Sweden.

This study was supported by the Swedish Research Council (12660), the Stockholm County Council, the Swedish Heart-Lung foundation, the Tornspiran Foundation, the Mats Kleberg Foundation, the European Commission (FAD, Health-F2-2008200647), and a donation by Fredrik Lundberg.

Disclosures: Authors have nothing to disclose with regard to commercial support.

Received for publication May 9, 2011; revisions received Aug 3, 2011; accepted for publication Aug 26, 2011; available ahead of print Sept 28, 2011.

Address for reprints: Veronica Jackson, MD, Cardiothoracic Surgery Unit, N3:03, Karolinska University Hospital, 17176 Stockholm, Sweden (E-mail: veronica. jackson@karolinska.se).

0022-5223/\$36.00

Copyright (c) 2012 by The American Association for Thoracic Surgery

doi:10.1016/j.jtcvs.2011.08.043 formation has also been put forward. In this context, proteolysis has been implicated as an important factor of the proposed aortopathy. The main hypothesis is that an altered turnover of extracellular matrix, mediated by matrix metalloproteinases (MMPs), is present in the aneurysmal aorta. ${ }^{5}$

Several different cell types have the capacity to express MMPs, for example, endothelial cells, leukocytes, macrophages, fibroblasts, and vascular smooth muscle cells (SMCs). There are more than 20 different known human MMPs and 4 endogenous tissue inhibitors of metalloproteinases (TIMPs). The tissue distribution and function of several of these enzymes are known to only a limited extent, ${ }^{6}$ but some clues as to what role MMPs and TIMPs may play in formation of thoracic aortic aneurysm (TAAs) has been presented. Previous work has mainly shown that increased expression of MMP2 and no significant alterations in MMP9, TIMP1, and TIMP2 expression is evident in TAA associated with BAV, but the results are conflicting. Some investigators have also positively correlated increased MMP2 expression in BAV patients to aortic diameter. ${ }^{7,8}$

The underlying molecular mechanisms of TAA formation in patients with bicuspid or tricuspid aortic valves (TAVs) are complex. This study analyzed the occurrence of all known MMPs, together capable of degrading all 


$$
\begin{aligned}
& \text { Abbreviations and Acronyms } \\
& \text { BAV = bicuspid aortic valve } \\
& \text { MMP }=\text { matrix metalloproteinase } \\
& \text { mRNA }=\text { messenger ribonucleic acid } \\
& \text { PC }=\text { principal component } \\
& \text { PCA }=\text { principal components analysis } \\
& \text { PCR }=\text { polymerase chain reaction } \\
& \text { RNA }=\text { ribonucleic acid } \\
& \text { SMC }=\text { smooth muscle cell } \\
& \text { TAA }=\text { thoracic aortic aneurysm } \\
& \text { TAV }=\text { tricuspid aortic valve } \\
& \text { TIMP }=\text { tissue inhibitors of metalloproteinase }
\end{aligned}
$$

components of the extracellular matrix, in ascending aortic aneurysms associated with BAV or TAV.

\section{METHODS \\ Patients}

The study population consisted of 109 patients with disease of the aortic valve and/or aorta who underwent open cardiac operations at the Cardiothoracic Surgery Unit at the Karolinska University Hospital, 2007 to 2009. The patients were divided into groups depending on valvular morphologic status and disease of the ascending aorta (TAV, $n=40$; BAV, $\mathrm{n}=69$; dilated aorta, $\mathrm{n}=68$; nondilated aorta, $\mathrm{n}=41$ ) (Table 1). The ascending aorta was considered to be nondilated at a diameter of $4.0 \mathrm{~cm}$ or less and dilated at a diameter of $4.5 \mathrm{~cm}$ or more; subjects with aortic dimensions of 4.1 to $4.4 \mathrm{~cm}$ were not included in the analyses. Patients who were found to have concomitant significant coronary artery disease at the time of inclusion were excluded from the study. The study was approved by the local ethics committee and patient informed consent was obtained.

\section{Ribonucleic Acid (RNA) Isolation}

Biopsy tissue was taken from the anterior (convex) part of the aorta, that is, the site of the aortotomy a few centimeters above the aortic valve. The tissue was separated into intima/media and adventitia by adventicectomy. Total RNA was isolated using Trizol (GIBCO-BRL Life Technologies, Gaithersburg, Md) and RNeasy Mini kit (Qiagen, Crawley, United Kingdom) including treatment with RNase-free DNase set (Qiagen) according to manufacturer's instructions. The quality of RNA was analyzed with an Agilent 2100 bioanalyzer (Agilent, Santa Clara, Calif) and quantity was measured by a NanoDrop (Thermo Scientific, Waltham, Mass).

\section{Gene Arrays}

The RNA samples were hybridized and scanned at the Karolinska Institutet microarray core facility. Affymetrix GeneChip Human Exon 1.0 ST arrays and protocols were used (Affymetrix, Santa Clara, Calif). The raw Cel files were preprocessed and $\log 2$ transformed using Robust Multichip Average normalization as implemented in the Affymetrix Power Tools 1.10.2 package apt-probeset-summarize. All investigations were done on the core sets of meta probes provided by Affymetrix. Cutoff level for gene expression was defined at 4.5, which was the level below which most Y-chromosome genes were found in female arrays.

\section{Real-time Polymerase Chain Reaction (PCR)}

Samples were analyzed in duplicates and evaluated with a standard curve using TaqMan Gene Expression Assay (Applied Biosystems, Carlsbad, Calif) using the following assays: MMP1 (Hs00233958), MMP2 (Hs00234422), MMP3 (Hs00233962), MMP7 (Hs00159163), MMP9 (Hs00957562), MMP12 (Hs00159178), MMP13 (Hs00233992), MMP14 (Hs00237119), MMP19 (Hs00275699), MMP21 (Hs01040378), MMP25 (Hs00360851), TIMP2 (Hs00234278), and TIMP4 (Hs00162784). The Ribosomal Protein Large P0 (RPLP0) (Hs99999902) was used as a housekeeping gene.

\section{Immunohistochemistry}

Aortic specimens were fixated in $4 \%$ zinc formaldehyde (Histolab AB, Gothenburg, Sweden) and embedded in paraffin. Sections $5-\mu \mathrm{m}$ thick were pretreated with DIVA decloaker $10 \times$ solution (Biocare Medical, Concord, Calif). Primary antibodies were rabbit monoclonal to MMP14 (1:25; Abcam, Cambridge, United Kingdom), rabbit polyclonal to MMP19 (1:100;

\begin{tabular}{|c|c|c|c|c|}
\hline \multirow[b]{2}{*}{ Aortic diameter } & \multicolumn{2}{|c|}{ TAV } & \multicolumn{2}{|c|}{ BAV } \\
\hline & $\leq 40 \mathrm{~mm}$ & $\geq 45 \mathrm{~mm}$ & $\leq \mathbf{4 0} \mathrm{mm}$ & $\geq 45 \mathrm{~mm}$ \\
\hline $\mathrm{N}$ & 17 & 23 & 24 & 45 \\
\hline Gender, No. of women & 5 & 9 & 5 & 10 \\
\hline Age $(y)$, mean \pm SD & $69 \pm 11$ & $61 \pm 15^{*}$ & $56 \pm 10$ & $60 \pm 12$ \\
\hline $\mathrm{BSA}\left(\mathrm{m}^{2}\right.$ mean $\pm \mathrm{SD}$ & $1.99 \pm 0.19$ & $1.97 \pm 0.25$ & $1.99 \pm 0.21$ & $2.02 \pm 0.20$ \\
\hline Max aortic diameter (mm) & $33.7 \pm 3.9$ & $53.8 \pm 7.6 \dagger$ & $36.1 \pm 3.1$ & $50.3 \pm 3.3 \dagger$ \\
\hline Mean $\pm \mathrm{SD}(\min , \max )$ & $(27.5,39.8)$ & $(45.0,70.0)$ & $(30.2,39.9)$ & $(45.1,57.1)$ \\
\hline Max aortic diameter/BSA $\left(\mathrm{mm} / \mathrm{m}^{2}\right)$ & $17 \pm 2$ & $28 \pm 7 \dagger$ & $18 \pm 2$ & $25 \pm 3 \dagger$ \\
\hline AS & 12 & $1 \ddagger$ & 13 & $20 \ddagger$ \\
\hline AR & 5 & 18 & 11 & 14 \\
\hline Normal aortic valve & 0 & 4 & 0 & 11 \\
\hline
\end{tabular}
Abcam, Cambridge, United Kingdom), mouse monoclonal to alpha smooth muscle actin (1:5000; Sigma-Aldrich, St Louis, Mo), mouse monoclonal to CD68 (1:100; Novocastra, Leica Microsystems, Wetzlar, Germany), and mouse monoclonal to von Willenbrand factor (1:5000; DAKO, Glostrup, Denmark).

\section{Statistical Analysis}

The statistical analysis was performed using Statview software (SAS Institute, Inc, Cary, NC). The Mann-Whitney $U$ test was used for groupwise comparisons and the Spearman rank correlation test for correlations

TABLE 1. Patient demographics

Values are presented as mean \pm standard deviation ( $S D)$ or frequency. $T A V$, Tricuspid aortic valve; $B A V$, bicuspid aortic valve; $B S A$, body surface area; $A S$, aortic stenosis; $A R$, aortic regurgitation. Nondilated versus dilated aorta: $* P=.04 . \dagger P<.0001$. $\ddagger$ Valvular pathology (AS vs AR vs normal) in nondilated versus dilated aorta: TAV $P<.0001 ; \mathrm{BAV}$ $P=.03$. 
analyses. The $\chi^{2}$ test was used to analyze frequencies. Values are given as mean with standard deviation or median with interquartile range when applicable.

In addition, principal components analysis (PCA) was performed on Affymetrix Human Exon 1.0 ST Array meta probe set level data that had been preprocessed using Robust Multichip Average normalization. Before PCA, the data were scaled to unit variance and mean centered. In the intima/media analysis, the first 3 principal components (PCs) were enough (according to cross-validation) to describe $63 \%, 64 \%$, and $67 \%$ of the variance $\left(\mathrm{R}_{\mathrm{x}}{ }^{2}\right)$ in the TAV and BAV together, TAV separately, and BAV separately data sets, respectively. The $\mathrm{Q}_{\mathrm{x}}{ }^{2}$ (cumulative fraction of the total variation of $\mathrm{X}$ that can be predicted by the model) parameter for the 3 models is according to the following: TAV and BAV together, 0.33; TAV separately, 0.24; and BAV separately, 0.36. Multivariate model quality parameters for adventitia models after 3 PCs are according to the following: TAV separately, $\mathrm{R}_{\mathrm{x}}{ }^{2}=0.66, \mathrm{Q}_{\mathrm{x}}{ }^{2}=0.049$; BAV separately, $\mathrm{R}_{\mathrm{x}}{ }^{2}=0.64, \mathrm{Q}_{\mathrm{x}}{ }^{2}=0.30 . \mathrm{PCA}$ was performed using the SIMCA P+12.0.1 $\times 64$ (Umetrics, Umeå, Sweden) software. The tolerance ellipsoid/ellipse based on Hotelling's T2 at a significance level of .05 is calculated and shown in the score plots.

\section{RESULTS}

\section{No mRNA Expression of Collagenases Detected in Ascending Aortic Wall}

We detected mRNA expression for gelatinases (MMP2 and MMP9), stromelysin 3 (MMP11), all membranebound MMPs (MMP14, MMP15, MMP16, MMP17, MMP24, and MMP25) and for MMP19, MMP21, and MMP28 in the intima/media section of nondilated aorta in patients with BAV and TAV (Table 2). There were no differences in mRNA expression in nondilated aorta between BAV and TAV patients (data not shown). No mRNA expression of the collagenases (MMP1, MMP8, and MMP13) could be detected. Furthermore, TIMP1 to TIMP4 were all expressed at levels above background (Table 2). The same expression pattern of MMPs and TIMPs was detected in both the intimal/medial (Table 2) and the adventitial layers of dilated aortic specimens (data not shown). To confirm the expression measurements of the exon arrays, we performed real-time PCR for a subset of MMPs and TIMPs. The results of the 2 different experimental methods were compared, and positive correlations were found between the results from the exon array analysis and the results of the real-time PCR analysis for all the MMPs tested except for MMP25 (TAV: MMP2 Rho 0.84, $P<.001$; MMP9 Rho 0.72, $P<.001$; MMP14 Rho 0.84, $P<.001 ;$ MMP19 Rho $0.57, P=.009$; MMP25 Rho $0.27, P>.2$; TIMP2 Rho $0.75, P<.001$; TIMP4 Rho 0.90, $P<.001$ ). MMP25 had expression levels just above our defined threshold in microarray measurements and similar low expression levels (cycle threshold values of 36) when analyzed with real-time PCR. Furthermore, the low mRNA expression of MMP1, MMP3, MMP7, MMP12, and MMP13 was confirmed using real-time PCR (mean cycle threshold values of 36, 36, 33, 33 , and 31 , respectively).

\section{The Gene Expression Pattern of MMPs and TIMPs in Ascending Aorta Differs According to Valvular Structure and Aortic Disease}

A PCA based on the expression of the identified MMPs and TIMPs in intima/media showed no separation of patients with dilated aortas from patients with nondilated aortas when analyzing BAV and TAV patients together (Figure 1, A) nor when analyzing BAV patients separately (Figure 1,C). However, TAV patients with dilated aortas could be separated from patients with nondilated aortas (Figure 1, B). This suggests that differences in mRNA expression of MMPs and TIMPs, in the intimal/medial layer, between dilated and nondilated aortas are mainly confined

TABLE 2. Log2 mRNA expression levels in intima/media of non-dilated and dilated ascending aorta

\begin{tabular}{|c|c|c|c|c|c|c|}
\hline & $\begin{array}{c}\text { TAV } \\
\text { Nondilated }\end{array}$ & $\begin{array}{c}\text { TAV } \\
\text { Dilated }\end{array}$ & $\begin{array}{c}\text { TAV } \\
P \text { value }\end{array}$ & $\begin{array}{c}\text { BAV } \\
\text { Nondilated }\end{array}$ & $\begin{array}{c}\text { BAV } \\
\text { Dilated }\end{array}$ & $\begin{array}{c}\text { BAV } \\
P \text { value } \\
\end{array}$ \\
\hline MMP2 & $10.06 \pm 0.54(9.20,10.90)$ & $10.13 \pm 1.00(9.14,11.24)$ & $>.2$ & $10.14 \pm 0.45(9.15,10.99)$ & $10.34 \pm 0.66(8.54,11.30)$ & .12 \\
\hline MMP9 & $6.00 \pm 0.51(5.37,7.03)$ & $6.09 \pm 0.63(5.45,9.22)$ & $>.2$ & $5.83 \pm 0.21(5.63,6.78)$ & $5.83 \pm 0.28(5.57,6.34)$ & $>.2$ \\
\hline MMP11 & $6.24 \pm 0.38(5.75,6.69)$ & $6.35 \pm 0.22(6.08,7.32)$ & .18 & $6.26 \pm 0.34(5.84,6.77)$ & $6.14 \pm 0.33(5.51,6.61)$ & $>.2$ \\
\hline MMP14 & $7.99 \pm 0.39(7.32,8.53)$ & $8.49 \pm 0.60(7.68,9.15)$ & $<.001$ & $8.01 \pm 0.51(7.32,8.78)$ & $8.31 \pm 0.48(6.52,8.90)$ & .009 \\
\hline MMP15 & $6.16 \pm 0.29(5.55,6.57)$ & $5.97 \pm 0.28(5.72,6.37)$ & .10 & $5.93 \pm 0.44(5.55,6.54)$ & $5.97 \pm 0.22(5.55,6.36)$ & $>.2$ \\
\hline MMP16 & $7.62 \pm 0.47(7.06,8.88)$ & $7.50 \pm 0.92(4.74,9.01)$ & $>.2$ & $7.91 \pm 0.80(6.54,8.87)$ & $7.81 \pm 0.58(6.71,8.97)$ & $>.2$ \\
\hline MMP17 & $6.07 \pm 0.48(5.39,6.52)$ & $5.94 \pm 0.32(5.51,6.28)$ & .022 & $6.04 \pm 0.56(5.29,6.55)$ & $5.93 \pm 0.38(5.45,6.45)$ & $>.2$ \\
\hline MMP19 & $5.60 \pm 0.41(4.97,6.28)$ & $5.90 \pm 0.49(5.52,7.34)$ & .001 & $5.57 \pm 0.51(5.03,6.15)$ & $5.79 \pm 0.37(5.23,6.35)$ & .010 \\
\hline MMP21 & $5.08 \pm 0.36(4.51,5.48)$ & $4.83 \pm 0.17(4.64,5.13)$ & .008 & $4.88 \pm 0.41(4.69,5.57)$ & $4.91 \pm 0.27(4.63,5.49)$ & $>.2$ \\
\hline MMP24 & $5.39 \pm 0.42(4.77,5.65)$ & $5.10 \pm 0.21(4.86,5.50)$ & .054 & $5.19 \pm 0.37(4.75,5.76)$ & $5.20 \pm 0.32(4.83,5.66)$ & $>.2$ \\
\hline MMP25 & $6.03 \pm 0.41(5.45,6.40)$ & $5.80 \pm 0.24(5.55,6.17)$ & .019 & $5.83 \pm 0.40(5.17,6.42)$ & $5.80 \pm 0.23(5.43,6.19)$ & $>.2$ \\
\hline MMP28 & $6.27 \pm 0.42(5.70,6.52)$ & $6.03 \pm 0.25(5.62,6.37)$ & .028 & $6.12 \pm 0.30(5.82,6.52)$ & $6.11 \pm 0.29(5.59,6.53)$ & $>.2$ \\
\hline TIMP1 & $11.95 \pm 0.51(11.51,12.87)$ & $11.82 \pm 0.69(11.14,13.29)$ & $>.2$ & $11.73 \pm 0.63(10.82,12.42)$ & $11.70 \pm 0.48(10.19,12.42)$ & $>.2$ \\
\hline TIMP2 & $11.25 \pm 0.32(10.41,12.01)$ & $11.58 \pm 0.47(11.13,12.09)$ & .007 & $11.32 \pm 0.52(10.60,12.12)$ & $11.76 \pm 0.61(9.84,12.35)$ & .001 \\
\hline TIMP3 & $8.38 \pm 1.19(7.76,10.09)$ & $8.99 \pm 0.67(8.18,10.73)$ & .18 & $9.23 \pm 0.76(8.05,10.12)$ & $9.10 \pm 0.44(7.35,10.26)$ & $>.2$ \\
\hline TIMP4 & $7.18 \pm 1.00(6.48,8.71)$ & $6.58 \pm 0.62(5.88,8.21)$ & $<.001$ & $7.13 \pm 0.85(6.12,9.03)$ & $6.96 \pm 0.75(5.93,8.40)$ & $>.2$ \\
\hline
\end{tabular}

Values are presented as median \pm interquartile range and minimum, maximum. $m R N A$, Messenger ribonucleic acid; $T A V$, tricuspid aortic valve; $B A V$, bicuspid aortic valve; $M M P$, matrix metalloproteinases; TIMP, tissue inhibitor of matrix metalloproteinases. 


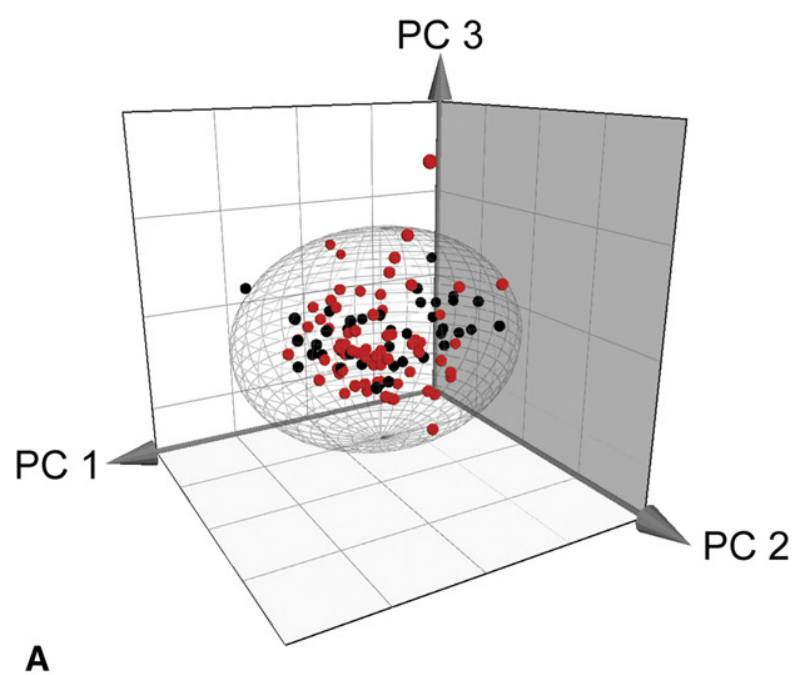

A

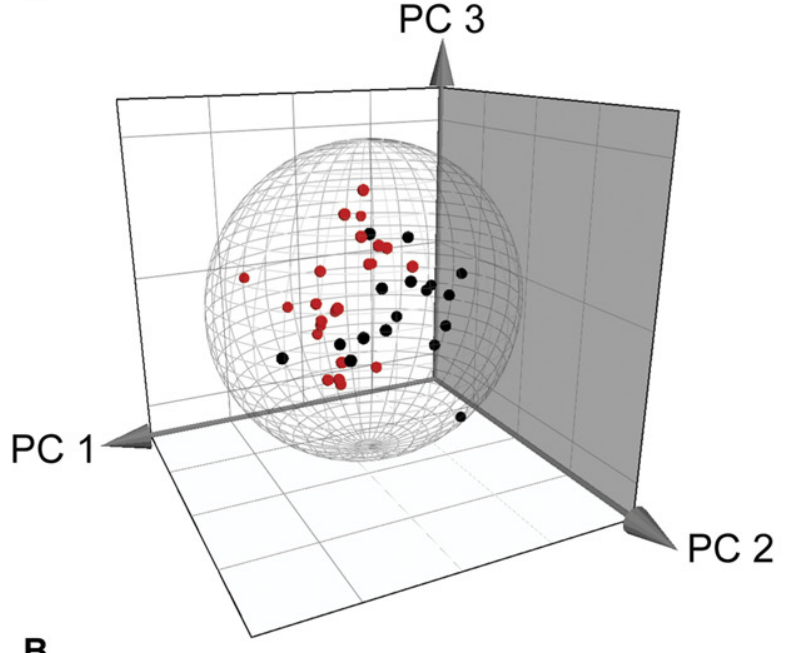

B

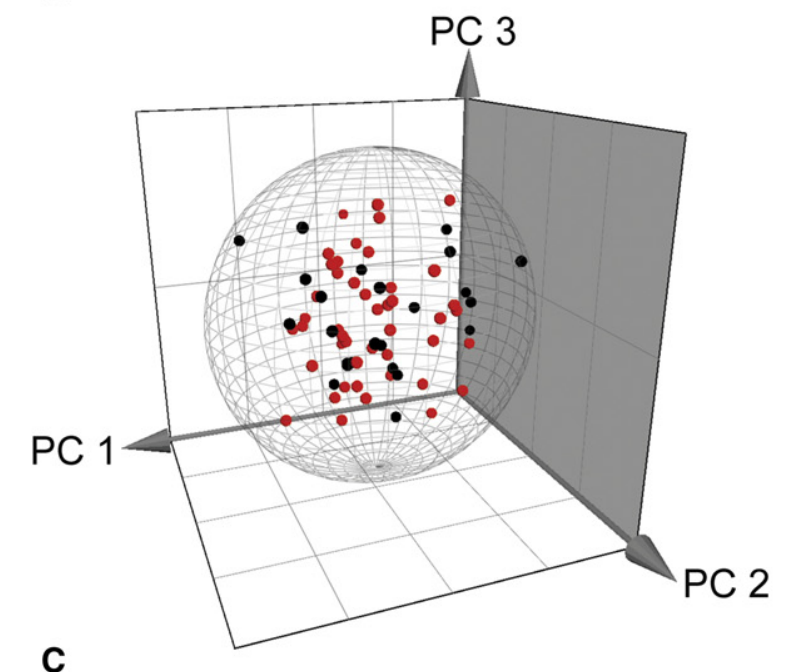

FIGURE 1. PCA of Affymetrix Human Exon 1.0 ST Array meta probe set level data of 12 MMP and 4 TIMP genes expressed in aortic samples from 109 patients. Three-dimensional score plots showing PC 1 to PC 3 plane of nondilated (black) and dilated (red) aortic samples, BAV and TAV together (A), TAV (B), and BAV (C). A PCA, in this case, is a projection to 3 to TAV patients. Figure 2, A, shows that the dilated aortas (red dots) of TAV patients are forming a cluster in the lower right quadrant of the multivariate score plot in the direction of first PC. PC 1 is thus responsible for separating dilated from nondilated aortic tissue. The genes responsible for this clustering of patients can be found in the lower right quadrant of the multivariate loading plot (MMP14, MMP19, MMP9, and TIMP3, Figure 2, $B$ ).

The same analysis was also performed on samples from adventitia giving rise to a good model in BAV, but the separation between dilated and nondilated tissue was not clear. The multivariate model in TAV, however, was not satisfying $\left(\mathrm{Q}_{\mathrm{x}}{ }^{2}=0.049\right)$, thereby showing no separation between dilated and nondilated tissue (analysis not shown).

Univariate analyses of differences in MMP and TIMP mRNA expression in the intima/media of TAV patients showed higher expression levels of MMP14, MMP19, and TIMP2 in dilated versus nondilated aortas (Table 2). In contrast, MMP17, MMP21, MMP25, MMP28, and TIMP4 all showed lower expression levels in dilated aortas in TAV patients. In BAV patients, MMP14, MMP19, and TIMP2 also showed higher expression levels in dilated aortas (Table 2). None of the MMPs or TIMPs was found to have lower expression levels in BAV patients with dilated versus nondilated aortas. There was no difference in MMP9 and TIMP3 expression in the intima/media section comparing dilated and nondilated aortas in TAV and BAV patients (Table 2).

To further analyze the intima/media of TAV patients with dilated aortas, we performed correlation analyses of MMP expression levels and maximal ascending aortic diameter measured $4 \mathrm{~cm}$ above the aortic valve. Of the MMPs that showed higher expression levels in TAV patients with dilated aortas, MMP19 correlated positively to maximal aortic diameter (Rho 0.61, $P=.004$, and Rho 0.57, $P=.008$ for raw and BSA-indexed values, respectively). MMP14 showed borderline significance for raw values (Rho 0.40, $P=.063)$ but did not correlate to BSA-indexed values (Rho $0.12, P>$.2). TIMP2 did not correlate to aortic diameter (Rho 0.26, $P=.23$, and Rho $0.02, P>.2$ ). Of the MMPs and TIMPs that showed lower expression levels in TAV patients with dilated aortas, only TIMP4 correlated negatively to raw values (Rho $-0.49, P=.021$ ) whereas MMP25, MMP28 and TIMP4 correlated negatively to BSA-indexed values (Rho $-0.45, P=.036$, Rho -0.43 ,

dimensions from 16 variables that are composed of the gene expression levels of the MMPs included in the study. Each patient will thereby be represented by a point in a 3-dimensional PC plane that summarizes the original 16 dimensional properties of the particular patient. $P C A$, Principal components analysis; $P C$, principal component; $B A V$, bicuspid aortic valve; $T A V$, tricuspid aortic valve; $M M P$, matrix metalloproteinase; TIMP, tissue inhibitors of metalloproteinase. 

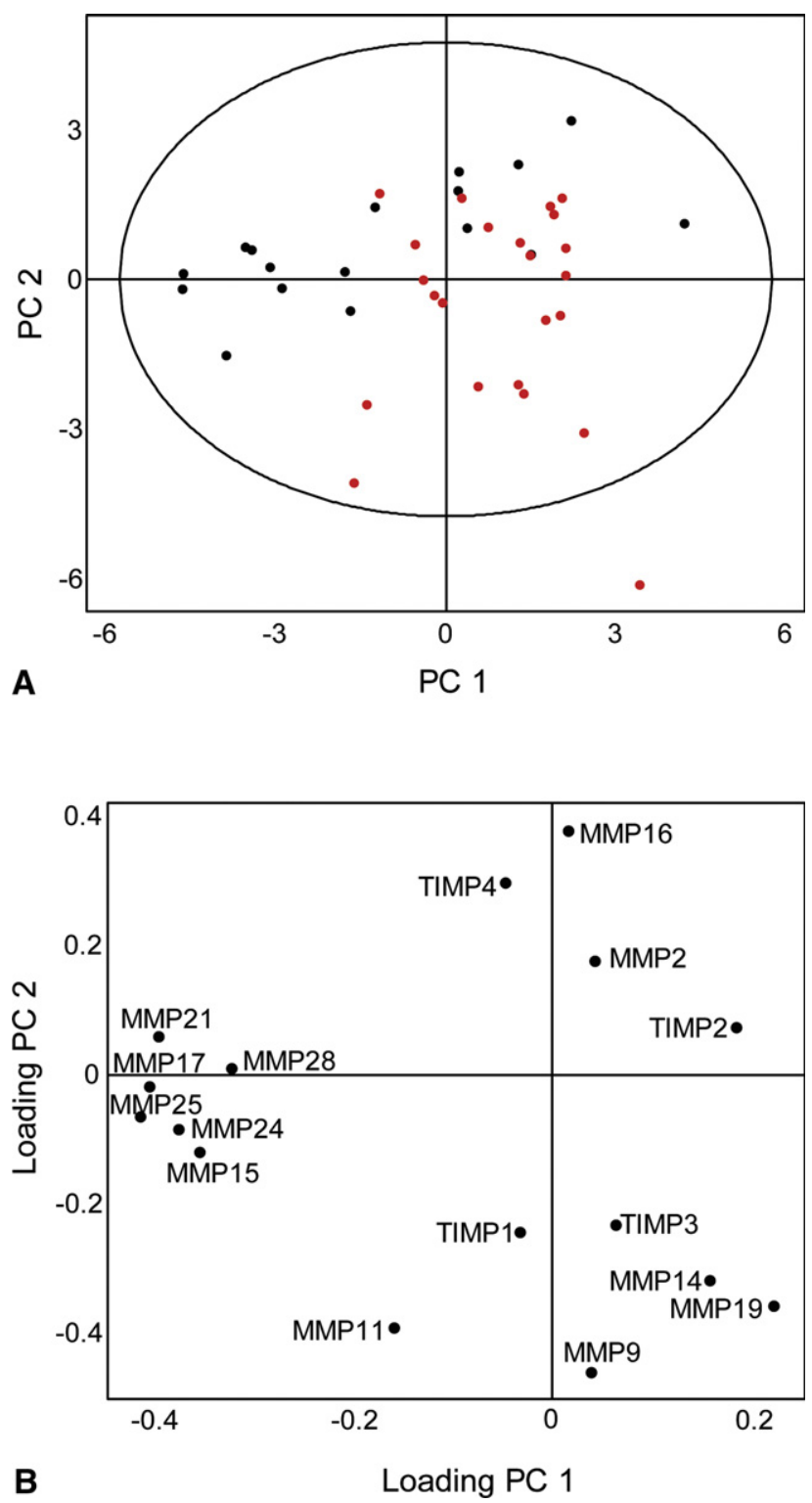

FIGURE 2. PCA of Affymetrix Human Exon 1.0 ST Array meta probe set level data of 12 MMP and 4 TIMP genes expressed in aortic samples from 40 patients with TAV. A, PC score plot showing nondilated (black) and dilated (red) aortic samples. B, Loading PC plot showing the corresponding genes (black dots) on meta probe set level. The loading plot shows which genes are responsible for clustering of the patients in the score plot. $P C A$, Principal components analysis; $P C$, principal component; $T A V$, tricuspid aortic valve; $M M P$, matrix metalloproteinase; TIMP, tissue inhibitors of metalloproteinase.

$P=.046$, and Rho $-0.55, P=.010$, respectively). In adventitia, MMP14 and MMP19 expression did not correlate to maximal aortic diameter in TAV patients (Rho -0.039 , $P>.2$, and Rho $0.16, P>.2$, respectively, data not shown). In BAV patients, MMP14, MMP19, and TIMP2 expression did not correlate to either raw maximal aortic diameter (Rho $0.008, P>.2$, Rho $-0.27, P>.2$, and Rho $0.20, P>.2$, respectively) or BSA-indexed values (Rho $-0.13, P>.2$, Rho $-0.20, P=.20$, and Rho $0.12, P>.2$, respectively).

\section{MMP14 and MMP19 Protein Expression Is Upregulated in the Medial Layer of Dilated Aorta}

Immunohistochemistry was used to localize the expression of MMP14 and MMP19 in aortic wall of TAV patients. The analysis showed that MMP19 protein was expressed mainly by endothelial cells (cells expressing von Willebrand factor) in patients with nondilated aortas (Figure 3). In dilated aortas, the endothelial expression was still present, but a strong MMP19 expression was also evident in the medial layer. Similar to MMP19, MMP14 protein expression was upregulated in the media of dilated aortas.

\section{DISCUSSION}

This study analyzed the mRNA expression of all known members of the MMP family in the ascending aortic wall to characterize protease expression in regard to valvular and aortic morphology. PCA of mRNA expression suggested that a change in MMP expression profile is especially pertinent in TAV patients with dilated aortas as compared with TAV patients with nondilated aortas. Furthermore, our study identified MMP14 and MMP19 as potentially important factors in the pathogenesis of TAA when TAV is present. The mRNA expression of MMP19 correlated with aortic diameter in TAV patients but not in BAV patients. Our data are in agreement with previous findings suggesting that the underlying mechanisms of BAV-associated TAA differs from TAV-associated TAA. ${ }^{9,10}$ Furthermore, we found significant gene expression levels for gelatinases, MMP11, all membranebound MMPs, and MMP19, MMP21, and MMP28. This finding is different from what is found for abdominal aortic aneurysms where MMP1, MMP2, MMP3, MMP8, MMP9, MMP12, MMP13, and MMP14 are considered to constitute the main contribution of the MMP family in aneurysm formation. ${ }^{11}$

Our findings support the idea that the pathogenesis of TAV- and BAV-associated TAA differs. When we were analyzing the differences in mRNA expression levels between dilated and nondilated aortas of TAV and BAV patients using a multivariate analysis, differences between the groups were mainly detected in aortas from TAV patients. There were no differences in mRNA expression between BAV and TAV in nondilated aortas. We then proceeded with analyses of the TAV group separate from the BAV group with special reference to differences between nondilated aortas and dilated aortas within the 2 groups. From this, MMP14, MMP19, and TIMP2 stood out as possibly upregulated in both TAV and BAV patients with dilated aortas, whereas possibly downregulated MMPs were found only in the TAV group. A correlation analysis between the mRNA expression of MMP14, MMP19, TIMP2, and 

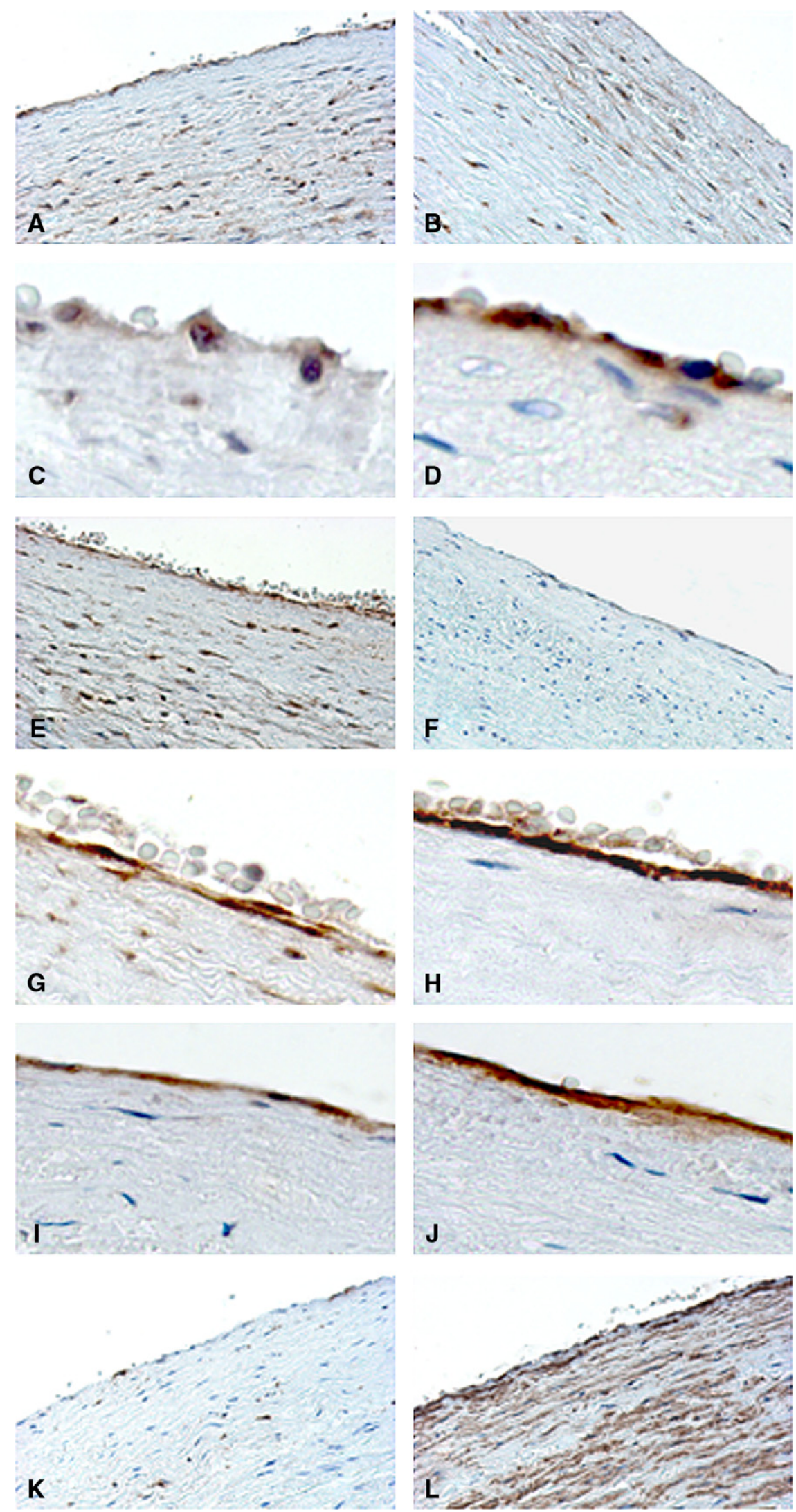
maximal aortic diameter revealed a strong positive correlation of MMP19 to maximal aortic diameter, but once again only in TAV patients. However, it should be stressed that the ranges in aortic diameters were narrower in BAV than in TAV patients, which could influence the analysis. The PCA showed a separation of nondilated and dilated specimens only in the TAV group, thus corroborating the results from the 2 preceding analyses, that is, that the MMP and TIMP profile in TAV patients with dilated aortas is likely to be important to the pathogenesis of TAA in these patients.

MMP19 showed a higher expression level in dilated aortas in comparison with nondilated aortas, and its expression correlated with aortic diameter. MMP19 can hydrolyze several matrix components such as collagen IV, laminin, nidogen, tenascin, fibronectin, and gelatin. ${ }^{12}$ MMP19 expression has been found to be present in various normal tissues with the exception of leukocytes. ${ }^{13}$ Our results confirm previous findings by Kolb and associates ${ }^{14}$ of MMP19 being expressed in the media of blood vessels. Furthermore, Djonov and colleagues ${ }^{15}$ have presented evidence of MMP19 expression in both SMCs and the endothelium in large and medium-sized blood vessels.

MMP14 is in its substrate specificity similar to the MMPs found to be important in abdominal aortic aneurysm formation, that is, the collagenases, matrilysins, and stromelysins, and in this context is considered to promote the invasion of inflammatory cells into the aortic wall. ${ }^{11,16}$ However, similar to MMP19, the immunohistochemical analyses clearly showed that MMP14 protein expression was mainly upregulated in the medial layer of the dilated aorta, a region consisting of SMCs/myofibroblasts and only a few inflammatory cells. MMP14 is known to be involved in the activation of proMMP2 by complex binding to TIMP2 ${ }^{17}$ In this study, TIMP2 showed higher expression levels in dilated versus nondilated aortas in both TAV and BAV patients in the univariate analysis. However, TIMP2 did not contribute to the group of MMPs and TIMPs that separated dilated and nondilated aortas into clusters in the PCA. In addition, TIMP2 expression did not correlate to maximal aortic diameter. Whether TIMP2 plays a role in MMP14 activation in this study population is unknown.

In contrast to our results, Ikonimidis and coworkers ${ }^{8}$ have shown protein expression of collagenases (MMP1, MMP8, and MMP13) and MMP7 in aneurysms of the ascending aorta, and Borges and associates ${ }^{18}$ have shown that mainly
MMP7 but also MMP3 are retained in areas of mucoid degeneration in the ascending aorta of patients with various TAA etiologies. Furthermore, Koullias and associates ${ }^{19}$ have shown increased levels of MMP1 and MMP9 protein expression in patients with TAA (including specimens from both the ascending and descending aorta as well as from aneurysms, intramural hemtaomas, and dissections) in comparison with controls. The discrepancies between these findings and our results could be attributed to the sensitivity of the exon array analyses used. However, the results of the real-time PCR analysis confirmed the very low mRNA expression levels of these MMPs. Importantly, mRNA expression does not necessarily equal protein expression and/or enzymatic activity. Also, the mRNA analysis is based on RNA preparations from large parts of the aorta and local expression cannot be excluded. We analyzed the aortic tissue with the medial layer separated from the adventitia inasmuch as it was hypothesized that the adventitial and medial layers differ in inflammatory profile. However, the same MMPs were expressed in the adventitia as compared with the intima/media. Another explanation to the discrepancy between protein and mRNA expression pattern could be that these proteases are not synthesized in the vessel wall and that their detection is the result of an entrapment of circulating proteases within the aortic wall.

It is possible that decreased expression of MMP17, MMP21, MMP25, and MMP28 in TAV-related TAA are markers of dedifferentiation of SMCs/fibroblasts and/or SMC/myofibroblast apoptosis. MMP21 is structurally similar to MMP25 and is found in various tissues and in cells such as fibroblasts and inflammatory cells. ${ }^{20,21}$ Interestingly, lower MMP21 expression has been suggested as a marker of dedifferentiated tumor cells. ${ }^{22}$ Similar to MMP21, MMP28 is downregulated in the setting of malignant tumors. ${ }^{23} \mathrm{An}$ other plausible activity of these proteases is their potential role in apoptosis, a common event associated with the dilated aorta. ${ }^{24}$ However, MMP21 expression was not associated with apoptosis of tumor cells. ${ }^{25}$ The exact roles of MMP21, MMP25, and MMP28 in TAA formation need further analyses.

One limitation of the present work is that the study is heterogeneous, being composed of patients eligible for intervention owing to surgical indications. The cohort thus varies in gender distribution, valvular disease, surgical timing, and, owing to the latter, also in aortic size at the time of surgery. Furthermore, it is known that patients

\footnotetext{
FIGURE 3. Immunohistochemistry analyses of MMP14 and MMP19 protein expression in the intima/medial layer of dilated and nondilated aortic specimens from TAV patients. A and B, MMP14 staining in dilated (A) and in nondilated aorta $(\mathrm{B})(\times 20)$. C and D, Enlargements of MMP14 and von Willebrand factor (endothelial cells) staining, respectively, in consecutive sections of dilated aorta. E and F, MMP19 staining in dilated (E) and nondilated aorta (F) ( $\times 20)$. G-J, Enlargements of MMP19 (G, I) and von Willebrand factor (H, J) staining in consecutive sections of dilated (G, H) and nondilated aorta (I, J). K and L, CD68 staining (inflammatory cells) in dilated aorta $(\mathrm{K})$ and $\alpha$-actin staining (smooth muscle cells) $(\mathrm{L})(\times 20)$. TAV, Tricuspid aortic valve; $M M P$, matrix metalloproteinase; TIMP, tissue inhibitors of metalloproteinase.
} 
with TAV and BAV present with manifest disease at different ages and stages of disease. In addition, possible temporal differences in gene expression are difficult to study in human beings, and to the best of our knowledge there are as yet no adequate animal models available. Taken together, these factors should be considered in interpreting the results.

In summary, the present study identifies MMP14 and MMP19 as proteolytic enzymes potentially involved in TAA formation in the ascending aorta of TAV patients. Our findings support the theory that the pathogenesis of BAV-associated TAA differs from TAV-associated TAA.

\section{References}

1. Ward C. Clinical significance of the bicuspid aortic valve. Heart. 2000;83:81-5.

2. Tadros TM, Klein MD, Shapira OM. Ascending aortic dilatation associated with bicuspid aortic valve: pathophysiology, molecular biology, and clinical implications. Circulation. 2009;119:880-90.

3. Guntheroth WG. A critical review of the American College of Cardiology/American Heart Association practice guidelines on bicuspid aortic valve with dilated ascending aorta. Am J Cardiol. 2008;102:107-10.

4. McKusick VA, Logue RB, Bahnson HT. Association of aortic valvular disease and cystic medial necrosis of the ascending aorta; report of four instances. Circulation. 1957;16:188-94.

5. Fedak PW, Verma S, David TE, Leask RL, Weisel RD, Butany J. Clinical and pathophysiological implications of a bicuspid aortic valve. Circulation. 2002; 106:900-4.

6. Raffetto JD, Khalil RA. Matrix metalloproteinases and their inhibitors in vascular remodeling and vascular disease. Biochem Pharmacol. 2008;75:346-59.

7. Fedak PW, de Sa MP, Verma S, Nili N, Kazemian P, Butany J, et al. Vascular matrix remodeling in patients with bicuspid aortic valve malformations: implications for aortic dilatation. J Thorac Cardiovasc Surg. 2003;126:797-806.

8. Ikonomidis JS, Jones JA, Barbour JR, Stroud RE, Clark LL, Kaplan BS, et al. Expression of matrix metalloproteinases and endogenous inhibitors within ascending aortic aneurysms of patients with bicuspid or tricuspid aortic valves. J Thorac Cardiovasc Surg. 2007;133:1028-36.

9. Jones JA, Stroud RE, Kaplan BS, Leone AM, Bavaria JE, Gorman JH 3rd, et al Differential protein kinase $\mathrm{C}$ isoform abundance in ascending aortic aneurysms from patients with bicuspid versus tricuspid aortic valves. Circulation. 2007; 116(11 Suppl):I144-9.

10. Della Corte A, Quarto C, Bancone C, Castaldo C, Di Meglio F, Nurzynka D, et al. Spatiotemporal patterns of smooth muscle cell changes in ascending aortic dilatation with bicuspid and tricuspid aortic valve stenosis: focus on cell-matrix signaling. J Thorac Cardiovasc Surg. 2008;135:8-18.
11. Rizas KD, Ippagunta N, Tilson MD III. Immune cells and molecular mediators in the pathogenesis of the abdominal aortic aneurysm. Cardiol Rev. 2009;17: 201-10.

12. Stracke JO, Hutton M, Stewart M, Pendás AM, Smith B, López-Otin C, et al. Biochemical characterization of the catalytic domain of human matrix metalloproteinase 19. Evidence for a role as a potent basement membrane degrading enzyme. $J$ Biol Chem. 2000;275:14809-16.

13. Murphy G, Knauper V, Cowell S, Hembry R, Stanton H, Butler G, et al. Evaluation of some newer matrix metalloproteinases. Ann N Y Acad Sci. 1999;878: 25-39.

14. Kolb C, Mauch S, Peter HH, Krawinkel U, Sedlacek R. The matrix metalloproteinase RASI-1 is expressed in synovial blood vessels of a rheumatoid arthritis patient. Immunol Lett. 1997;57:83-8.

15. Djonov V, Hogger K, Sedlacek R, Laissue J, Draeger A. MMP-19: cellular localization of a novel metalloproteinase within normal breast tissue and mammary gland tumours. J Pathol. 2001;195:147-55.

16. Takino T, Miyamori H, Watanabe Y, Yoshioka K, Seiki M, Sato H. Membrane type 1 matrix metalloproteinase regulates collagen-dependent mitogen-activated protein/extracellular signal-related kinase activation and cell migration. Cancer Res. 2004;64:1044-9.

17. Klein T, Bischoff R. Physiology and pathophysiology of matrix metalloproteases. Amino Acids. 2011;41:271-90.

18. Borges LF, Touat Z, Leclercq A, Zen AA, Jondeau' G, Franc B, et al. Tissue diffusion and retention of metalloproteinases in ascending aortic aneurysms and dissections. Hum Pathol. 2009;40:306-13.

19. Koullias GJ, Ravichandran P, Korkolis DP, Rimm DL, Elefteriades JA. Increased tissue microarray matrix metalloproteinase expression favors proteolysis in thoracic aortic aneurysms and dissections. Ann Thorac Surg. 2004;78:2106-10; discussion 2110-1

20. Ahokas K, Lohi J, Lohi H, Elomaa O, Karjalainen-Lindsberg ML, Kere J, et al. Matrix metalloproteinase-21, the human orthologue for XMMP, is expressed during fetal development and in cancer. Gene. 2002;301:31-41.

21. Skoog T, Ahokas K, Orsmark C, Jeskanen L, Isaka K, Saarialho-Kere U. MMP21 is expressed by macrophages and fibroblasts in vivo and in culture. Exp Dermatol. 2006;15:775-83.

22. Kuivanen T, Ahokas K, Virolainen S, Jahkola T, Hölttä E, Saksela O, et al. MMP21 is upregulated at early stages of melanoma progression but disappears with more aggressive phenotype. Virchows Arch. 2005;447:954-60.

23. Bister VO, Salmela MT, Karjalainen-Lindsberg ML, Uria J, Lohi J, Puolakkainen P, et al. Differential expression of three matrix metalloproteinases, MMP-19, MMP-26, and MMP-28, in normal and inflamed intestine and colon cancer. Dig Dis Sci. 2004;49:653-61.

24. Bonderman D, Gharehbaghi-Schnell E, Wollenek G, Maurer G, Baumgartner H, Lang IM. Mechanisms underlying aortic dilatation in congenital aortic valve malformation. Circulation. 1999;99:2138-43.

25. Ahokas K, Lohi J, Illman SA, Llano E, Elomaa O, Impola U, et al. Matrix metalloproteinase-21 is expressed epithelially during development and in cancer and is up-regulated by transforming growth factor-beta1 in keratinocytes. $\mathrm{Lab}$ Invest. 2003;83:1887-99. 